International Conference on Renewable Energies and Power Quality (ICREPQ'11)

Las Palmas de Gran Canaria (Spain), 13th to 15th April, 2011

\title{
Effects of non-zero phase harmonics on inductions machines and coupled mechanical loads
}

\author{
Filipe Oliveira ${ }^{1}$, Gerardo Peláez ${ }^{2}$, Manuel P. Donsión ${ }^{3}$, J. Iwaszkiewicz $^{4}$, J. Perz $^{4}$ \\ ${ }^{1}$ Department of Electrical Engineering. School of Technology and \\ Management. Polytechnic Institute of Leiria. Portugal \\ Institute for Systems and Computer Engineering at Coimbra. Portugal \\ ${ }^{2}$ Department of Mechanical Engineering. University of Vigo. Spain \\ ${ }^{3}$ Department of Electrical Engineering. University of Vigo. Spain \\ ${ }^{4}$ The Electrotechnical Institute. Gdansk Branch. Poland
}

\begin{abstract}
The presence and effect of harmonics in power systems is widely known, but some of its effects are still only partially studied and addressed in existing works. Traditionally, power system harmonics were nearly always not phase-shifted from the fundamental sine wave, and therefore the effect of the phase was not considered. This paper will address the influence of such harmonic content, with non-zero phase, in the behaviour of an induction machine and its mechanical load, namely in mechanical vibrations produced and speed stability. While mechanical vibrations are intrinsically present on rotating machines, both electrical and mechanical parameters can have a strong influence in their severity. This being a broad topic of research, this paper intends to show a preliminary approach destined to validate some of the starting points set for the development of further work.
\end{abstract}

\section{Key words}

Rotating machinery, power system harmonics, phase harmonics, mechanical

\section{Introduction}

It is well known that most electric motors, namely induction machines, are designed to operate in the linear portion of the hysteresis curve, and therefore the machine working parameters selected and the magnetic materials used are so calculated.

This, combined with the high time constants (in comparison with electric systems) of mechanical systems should also mean that higher frequency signals, such as those generated when the machine is driven by a variable speed drive, or fed with a harmonically rich network voltage, would produce little or no mechanical effects, due to the fact they would rest in the saturation part of the hysteresis curve.
However, it is well known, and stated by many authors, including the authors of this paper, that under conditions such as harmonics and PWM-modulation waves, mechanical aspects such as vibration and speed stability are affected. On the other hand, most studies address harmonic levels considering only each harmonic order in magnitude, but neglecting the effect that the phase of harmonic component has on the resulting voltage and current waveforms, and consequently on the behaviour of the electric machine and the coupled load.

This, of course, has much to do with the fact that traditionally voltage harmonics were mainly generated by non-linear loads and unloaded power transformers, which meant harmonics with non-zero phase were rare.

However, nowadays, considering the combined effect of the profusion of numerous non-linear loads, the decrease of linear loads, and the growing importance of power quality disturbances introduced by distributed generation, non-zero phase harmonics are becoming more present in power systems.

The main mathematical tool used to study a periodic signal, providing it as an infinite sum of sine waves, the Fourier transform, works with complex numbers, and this, of course, is not a mere curiosity - though most of the times, only the modulus is considered, the phase of the complex number provides the angle distance between the fundamental component, and each harmonic component.

In this paper, the issue of non-zero phase harmonics in the voltage wave feeding an induction motor, and its effects, will be addressed, mainly based on experimental trials conducted using a trial bench, a programmable power source and adequate measurement and acquisition equipment. The main ideas and conclusions will be presented, highlighting that phase, as well as magnitude, 
is of importance when characterizing the harmonic spectrum of a voltage wave, and its effects on the devices supplied by it.

\section{Voltage Waveform with phase harmonics}

Usually, harmonics in power systems are quantified either as a whole, using the Total Harmonic Distortion index, which is a pondered average of the magnitude of a number of harmonics, or individually, identifying each harmonic by its order and magnitude. Under this traditional classification, the phase of each harmonic is neglected, or considered to be null, which causes very different waveforms to be considered equal in harmonic content, and effects on end-user equipments.

As an example, let us consider a standard sine wave and one with a third harmonic of $20 \%$, at various phase angles. Figure 1 depicts such situation:

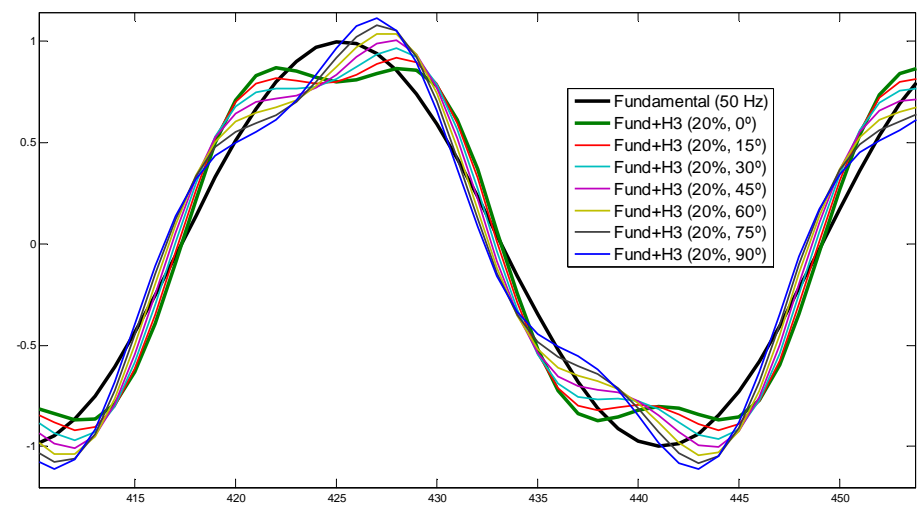

Fig. 1. Influence of the phase of the harmonic in the resulting waveform.

As is visible, the black and green waveforms are the well known pure sine wave, and one with a presence of the third harmonic at $0^{\circ}$, resulting that the 'hilltop' and the 'valley' of the sine wave are 'doubled' due to it. However, as the phase is shifted, the resulting waveform, depicted in the remaining waveforms in the figure, is considerably altered, even if all correspond to a sine wave with a $20 \%$ third harmonic.

Such facts lead to suspect that the behaviour of an electric machine may be somewhat different when fed with each of the waveforms in fig. 1. The following sections of this paper will present a summary of the results and conclusions from a set of experimental trials dedicated to further explore this question.

\section{Laboratory equipment}

In order to study the influence of the electric supply to the motor on the overall behaviour of a mechanical load, a specially designed, dimensioned and built one-stage inertia flexible rotor (OSIFRO) set was used. This provided a simple mechanical load with a well-fitted (and wellknown) dynamic model with which to work.

Fig. 2 shows a view of the OSIFRO model:

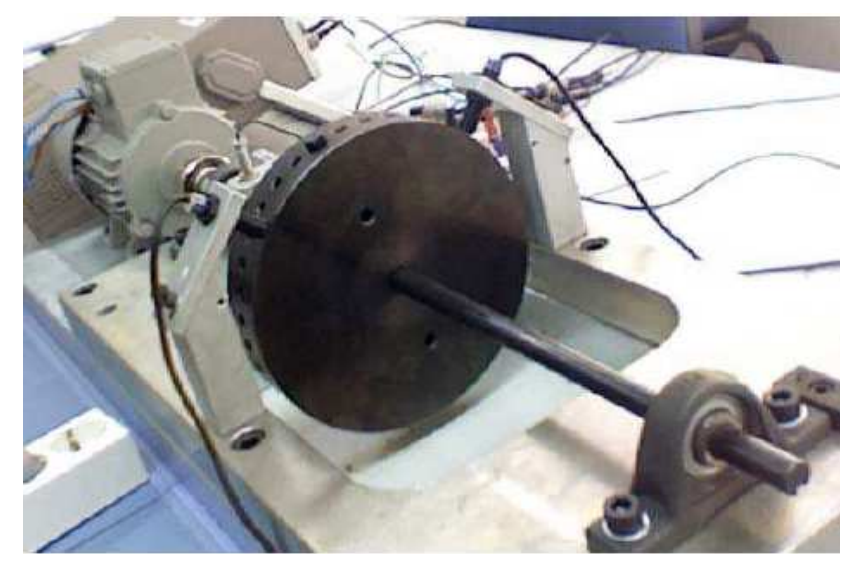

Fig. 2. A view of the OSIFRO

To drive the OSIFRO, a $0,37 \mathrm{~kW}$ squirrel-cage induction machine was connected using a three-phase input/output, $15 \mathrm{kVA}$ programmable AC power source from California Instruments. This power source provided all necessary waveforms, from perfect voltage sine waves of a range of amplitudes and frequencies to harmonically rich waveforms, programming each harmonic in magnitude and phase.

The OSIFRO mechanical load is constituted by a flexible, cylindrical rotor with a cylindrical inertia. This mechanical load is intrinsically slightly unbalanced, and adequate correction masses can be added to balance the rotor.

This work requires a number of electrical and mechanical quantities to be measured. In order to acquire and accommodate the several quantities to be measured, a PC-based, high-precision data acquisition card was used, along with voltage and current active probes, accelerometers, proximity inductive sensors and a keyphasor.

\section{Experimental trials and results}

In order to study, under laboratory conditions, the topics presented in the previous sections, a number of trial situations was devised and those trials were conducted in order to provide a preliminary approach to the question of the influence of the phases of the harmonic content on the set composed by the driven electric machine and its shaft and load.

The set was thus fed with waveforms containing different harmonic content and different phases, and the various significant quantities were acquired and recorded, and later analysed; a summary of this is presented in the following lines. 
A first set of trials included the pure sine wave (for standard reference) and combinations of the presence of the $3^{\text {rd }}$ harmonic in different percentage magnitudes and phases.

In these trials, mechanical vibrations are measured as the shaft displacement from the rest position, by means of two proximity sensors ( $\mathrm{x}$ and $\mathrm{y}$ ) installed in a $90^{\circ}$ angle, as depicted in Fig. 2.

Table 1 shows, in a summarized form, some of the results obtained concerning the level of shaft displacement under different harmonic levels:

\begin{tabular}{|l|c|c|c|c|}
\hline $\begin{array}{l}\text { Vibration } \\
\text { amplitude }\end{array}$ & $\begin{array}{l}\text { Amp x } \\
\text { (m.u.) }\end{array}$ & $\begin{array}{l}\text { Amp y } \\
-8 \mathrm{~m} . u .)\end{array}$ & $\begin{array}{l}\text { Quad. } \\
\text { measure }\end{array}$ & \% Dif \\
\hline Sine wave & 1,3293 & 1,0390 & 0,7023 & - \\
\hline $\begin{array}{l}\text { Sine }+20 \% \\
3^{\text {rd }} \text { harmonic } \\
\left(0^{\circ}\right)\end{array}$ & 1,3515 & 1,0648 & 0,7220 & $+2,81 \%$ \\
\hline $\begin{array}{l}\text { Sine }+20 \% \\
3^{\text {rd }} \text { harmonic } \\
\left(30^{\circ}\right)\end{array}$ & 1,3873 & 1,0992 & 0,7241 & $+3,10 \%$ \\
\hline
\end{tabular}

Table I - Vibration amplitude of the shaft under different harmonic content.

Although the measurement units indicated in the table do not translate into standard length units, they are coherent, and therefore the relative results are comparable.

Figure 3 depicts the waveforms generated by the distance to the axis as measured by sensor $\mathrm{x}$ and $\mathrm{y}$, and the quadrature radial distance vector modulus calculated from the two.

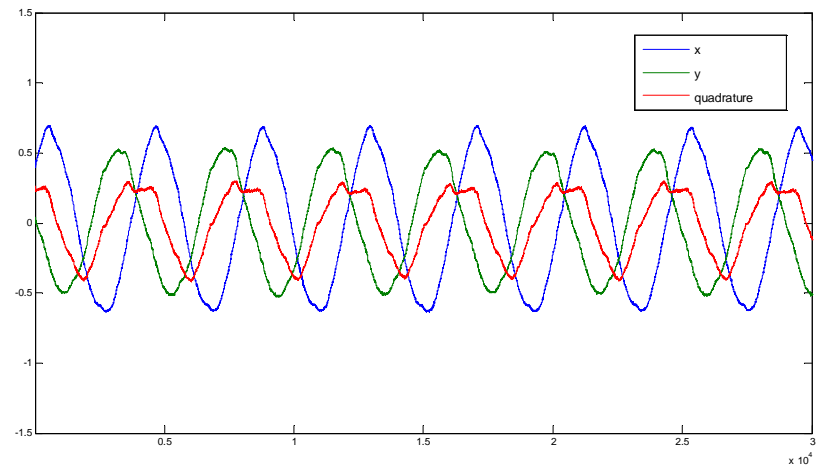

Fig. 3 - Measured vibration waveform in sensors $x, y$ and the radial distance calculated from the two for the waveform with $20 \% 3^{\text {rd }}$ Harmonic $\left(0^{\circ}\right)$.

It is visible from these results that the vibrations level increases when the $3^{\text {rd }}$ harmonic is introduced, and even more so when the $60^{\circ}$ phase angle is added. The additional vibration, in percentage, is, nevertheless, not very significant with this harmonic.

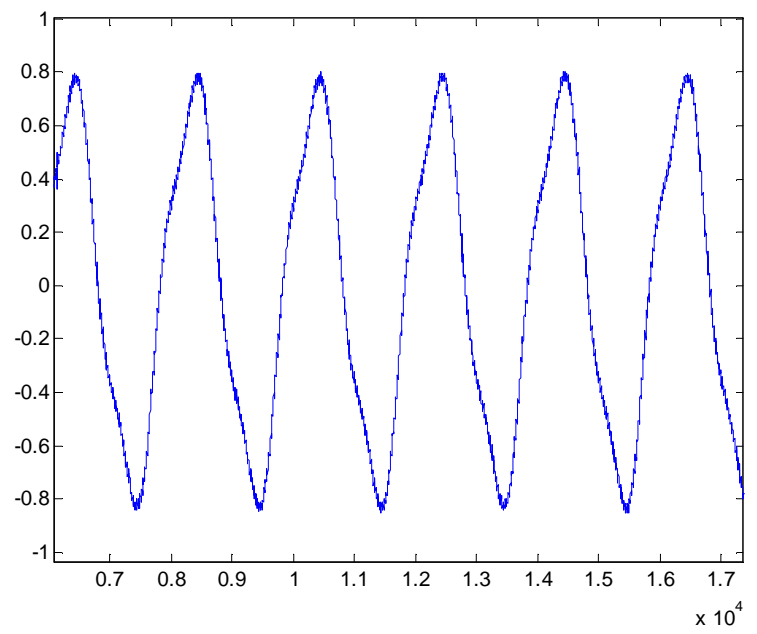

Fig. 4 - Current waveform produced in the motor when fed with the $20 \% 3^{\text {rd }}$ harmonic, $60^{\circ}$ phase voltage waveform.

Fig. 4 depicts the current waveform in the motor when the voltage waveform contains $20 \%$ third harmonic at a $60^{\circ}$ phase angle.

On the topic of speed stability, the results are slightly different and somewhat surprising. Table II and Fig. 5 present the main results from these measurements:

\begin{tabular}{|l|c|c|c|c|}
\hline $\begin{array}{l}\text { Speed } \\
\text { stability (Hz) }\end{array}$ & Mean & $\begin{array}{l}\% \text { Dif. } \\
\text { mean }\end{array}$ & $\begin{array}{l}\text { Std. } \\
\text { Dev. }\end{array}$ & $\begin{array}{l}\% \text { Dif } \\
\text { Std. Dev }\end{array}$ \\
\hline Sine wave & 24,1776 & - & 0,0981 & - \\
\hline $\begin{array}{l}\text { Sine }+20 \% \\
3^{\text {rd }} \text { H. }\left(0^{\circ}\right)\end{array}$ & 24,2064 & $+0,12 \%$ & 0,0387 & $-39,45 \%$ \\
\hline $\begin{array}{l}\text { Sine+ 20\% } 3^{\text {rd }} \\
\text { H. }\left(30^{\circ}\right)\end{array}$ & 24,2390 & $+0,25 \%$ & 0,1352 & $+37,89 \%$ \\
\hline
\end{tabular}

Table II - Speed stability of the shaft under different harmonic content.

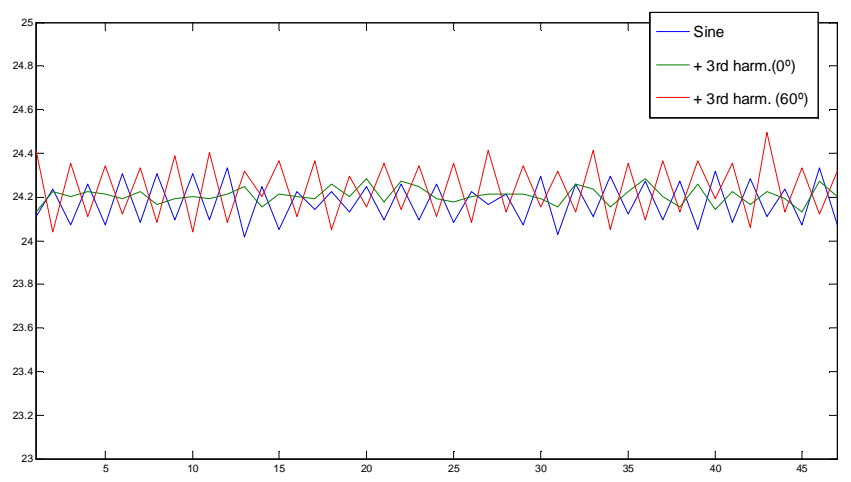

Fig. 5 - Speed stability of the shaft under different harmonic content of the voltage waveform

It is visible from the table and chart that, despite higher vibration measured as in Table I, the waveform with $20 \%$ $3^{\text {rd }}$ harmonics at $0^{\circ}$ presents a more stable rotational speed than the other combinations.

On the other hand, the same harmonic, but at $60^{\circ}$ phase angle, causes the speed stability to decrease considerably. This shows that more severe does not necessarily mean a more unstable rotational speed. 


\section{Conclusions and Outlook}

The immediate results from the laboratory experiments, here presented, suffice in the justification of the need for further study of the effects of the phase of the harmonic content on the overall effect of the harmonic content on the consumer equipments.

The combined effects of the harmonic content, the magnetic behaviour of the induction machine component materials and the mechanical characteristics of the load prove to be more complex than the approaches usually taken to deal with this matter.

Thus, further studies, including more laboratory trials, complete and adequate characterization of the materials, correct and thorough modelling of the mechanical and electromagnetic effects (including a more complex modelling of the hysteresis curve) are all needed if precise prediction of the behaviour of these and similar machines is to be achieved.

The application of laboratory trials rather than computational simulation is an added value of this contribution, since its results go sharply to the areas where simulation tends to fail, by not including, due to added complexity, all necessary parameters needed to adequately represent and predict the behaviour of a apparently simple, yet reasonably complex, electromagnetic-mechanical set, in the multiple relevant domains of physics.

Future work may then include any or many of these aspects, further exploring this topic starting from the preliminary paper.

\section{Acknowledgement}

The authors wish to acknowledge the financial support of Spanish government through "Ministerio de Ciencia e Innovación" and FEDER Funds. Research project ENE2007-6803-C04-01.

\section{References}

[1] R. Yacamini and S. C. Chang," Noise and Vibration from Induction Machines Fed from Harmonic Sources", IEEE Transactions on Energy Conversion, Vol. 10, No. 2, June 1995

[2] J.P. Den Hartog, "Mechanical Vibrations", MacGraw-Hill 4th ed. New York, 1956 (Reprint).

[3] Ching-Yin Lee (et. al), "Effects of Voltage Harmonics on the Electrical and Mechanical Performance of a Three-phase Induction Motor", IEEE, 1998

[4] F. Oliveira, M. P. Donsión, J. Iwaszkiewicz, J. Perz, "Rotating speed stability and mechanical vibrations analysis of a one-stage inertia flexible rotor driven by variable speed drives", Proceedings of ICREPQ'10 - International Conference on Renewable Energy and Power Quality, Granada (Spain), 2010

[5] F. Oliveira, M. P. Donsión, G. Peláez, "Dynamics parameters estimation of an asynchronous machine plus mechanical shaft set through orbit frequency response analysis", ICREPQ'10 - International Conference on Renewable Energy and Power Quality, Granada (Spain), 2010

[6] A. E. Fitzgerald et al, "Electric Machinery", John Wiley and sons, 6th edition, 2002 